\title{
LAS COMUNICACIONES INTEGRADAS DE MARKETING (CIM) COMO PILAR DE LA ESTRATEGIA DE MARKETING VERDE Y SUS IMPLICACIONES EN LA GESTIÓN AMBIENTAL*
}

\author{
NELCY ROCÍO ESCOBAR MORENO*** \\ UNIVERSIDAD NACIONAL DE COLOMBIA
}

Recibido/ Received/ Recebido: 12/03/2012 - Aceptado/ Accepted / Aprovado: 15/10/2012

\section{Resumen}

\begin{abstract}
Este documento examina el constructo de Comunicaciones Integradas de Marketing (CIM), desde su definición hasta su aplicación a partir de la perspectiva del mercadeo verde en la estrategia organizacional. El texto presenta tres grandes discusiones, primero, una reflexión particular sobre los aportes e implicaciones más importantes de las Comunicaciones Integradas de Marketing y de cada uno de sus instrumentos a la estrategia de gestión ambiental de las organizaciones. Luego, una caracterización general del consumidor verde y de otros stakeholders de la organización, en particular, de su papel en el proceso estratégico de la comunicación verde. Finalmente, como conclusión de este trabajo, se plantean algunas consideraciones sobre la necesaria aplicación de este concepto en la propuesta de gestión ambiental organizacional en la actualidad.
\end{abstract}

Palabras clave: Comunicaciones integradas de marketing, Comunicación verde, Marketing verde, Consumidor verde, Gestión ambiental.

\section{INTEGRATED MARKETING COMMUNICATIONS (IMC) AS AN IMPORTANT ISSUE IN GREEN MARKETING STRATEGY AND ITS IMPLICATIONS ON ENVIRONMENTAL MANAGEMENT}

\begin{abstract}
This document presents the structure of Integrated Marketing Communications, since its definition up to its application from green marketing approach in organizational strategy. The paper presents three major discussions, first, a particular reflection on contributions and most important implications of Integrated Marketing Communications and each one of its instruments to corporation's environmental management strategy. Then, it presents a general characterization of green consumer and other corporation's stakeholders, particularly, on its role during green communication strategic process. Finally, as a conclusion of this paper, there are some considerations on the necessary implementation of this concept in the current proposal of organizational environmental management. Keywords: Integrated marketing communications, Green communication, Green marketing, Green consumer, Environmental management.
\end{abstract}

\footnotetext{
* El artículo de reflexión obtenido como resultado parcial de la fase de evaluación del estado del arte del constructo Comunicaciones Integradas de Marketing (CIM), que se desarrolló para el proyecto de investigación "Caracterización y Evaluación preliminar de los Factores Determinantes de un Programa de Comunicaciones Integradas desde el Mercadeo para una Organización No Lucrativa: El Caso de la Universidad Nacional de Colombia".

** Docente becaria de la Facultad de Ciencias Económicas de la Universidad Nacional de Colombia. Joven Investigadora de la Escuela de Administración de Empresas y Contaduría Pública de la Universidad Nacional de Colombia. Administradora de Empresas y Magíster en Administración de la Universidad Nacional de Colombia. Integrante del Grupo de Investigación en Gestión y Organizaciones GRIEGO de la Universidad Nacional de Colombia. Correo electrónico: nrescobarm@unal.edu.co
} 


\title{
AS COMUNICAÇÕES INTEGRADAS DE MARKETING (CIM) COMO PILAR DA ESTRATÉGIA DE MARKETING VERDE E SUAS IMPLICAÇÕES NA GESTÃO AMBIENTAL
}

\begin{abstract}
Resumo
Este documento examina a estrutura das Comunicações Integradas de Marketing (CIM), da sua definição até sua aplicação a partir da perspectiva do marketing verde na estratégia organizacional. O texto apresenta três grandes discussões: em primeiro lugar, faz uma reflexão particular sobre as contribuições e as implicações mais importantes das Comunicações Integradas de Marketing e de cada um de seus instrumentos com a estratégia da gestão ambiental das organizações. Depois, uma caracterização geral do consumidor verde e de outros stakeholders da organização, em particular, sobre seu papel no processo estratégico da comunicação verde. Finalmente, como conclusões deste trabalho são feitas algumas considerações sobre a necessária aplicação deste conceito na proposta de gestão ambiental organizacional da atualidade.

Palavras chave: Comunicações integradas de marketing, Comunicação verde, Marketing verde, Consumidor verde, Gestão ambiental.

Escobar, N. (2012) Las Comunicaciones Integradas de Marketing (CIM) como pilar de la estrategia de marketing verde y sus implicaciones en la gestión ambiental. En: Revista de la Facultad de Ciencias Económicas de la Universidad Militar Nueva Granada. rev.fac.cienc.econ, XX (2).
\end{abstract}

JEL: M10, M31, M37, Q57.

\section{Introducción}

Diversos Investigadores destacados como Schultz (1991), Keegan et al. (1992), Duncan \& Everett (1993), Hartley \& Pickton (1999), Kotler et al. (1999), Schultz \& Kitchen, (2000), Low (2000), Duncan (2002), Swain (2004), Schultz \& Schultz (2004), Madhavaram et al. (2005), y Kliatchko (2005, 2008), han comenzado a plantear la necesidad de considerar al interior de las organizaciones modelos que permitan guiar la manera de tomar las decisiones referentes a las Comunicaciones de Marketing. Todos ellos resaltan la importancia de crear marcos conceptuales y métodos que proporcionen un enfoque completo y disciplinado a este proceso de toma de decisiones.

En este contexto, las organizaciones de hoy se cuestionan sobre qué actividades de comunicaciones en marketing permitirán influir en las percepciones, preferencias, y decisiones de sus consumidores de manera efectiva.
A esta situación no resultan ajenas las organizaciones cuya estrategia está enfocada en el ámbito ambiental, y dentro de las cuales por lo tanto se torne necesario gestionar actividades de marketing y programas que permitan alcanzar este objetivo de influencia en los consumidores, ahora ecológicos.

De acuerdo con Vélez (2001), el mercadeo ecológico en el ámbito mundial ha venido creciendo ostensiblemente. Las tendencias del mercadeo ecológico en el ámbito nacional e internacional, se están expandiendo hacia la generación de productos y servicios derivados de la biodiversidad y del avance de la biotecnología. Las causas que motivan éstas nuevas tendencias, están relacionadas con la necesidad emergente de las empresas por reciclar sus productos y renovar el interés de los consumidores por productos ecológicos. De allí que las empresas deben dirigir el mercadeo verde a través de un líder que involucre a todas las áreas de la organización y que vea en esta estrategia una oportunidad de crecimiento importante para la misma. Por tal motivo, se genera 
un interés en la investigación acerca de las necesidades de comunicación de los eco-consumidores; al igual que del reconocimiento de la incidencia de los demás stakeholders de la organización en las decisiones que se tomen con respecto al tema ambiental dentro de ella.

En este trabajo se hace una revisión sobre estudios teóricos del mundo académico, con miras a aportar una visión teórica e integrada de los principales conceptos que comprenden la gestión de Comunicaciones Integradas de Marketing (CIM), y en particular, cuál es su utilidad dentro de las estrategias de marketing verde de las organizaciones. Se sustenta bajo el reconocimiento de la importancia que tiene el análisis de las necesidades de comunicación del cliente ecológico, la evaluación de la incidencia de los stakeholders en la toma de decisiones de gestión ambiental de la organización, y la importancia que tiene la difusión, a través de la comunicación verde de las decisiones de gestión ambiental tomadas por la organización para las distintas audiencias que responden a su estrategia ambiental.

\section{Metodología}

Este artículo surge como parte de los resultados parciales de una revisión cualitativa de los modelos de CIM, desarrollado como parte de las investigaciones en la Maestría en Administración cursada actualmente por la autora. En este trabajo particularmente se presenta una revisión corta y genérica sobre el tema, no obstante se hace especial referencia a las aplicaciones de esta temática al interior de las estrategias de gestión ambiental organizacional, con la intención de descubrir los avances más recientes de un enfoque particular de aplicación de la CIM.

La primera parte del trabajo es de tipo descriptivo, y contiene una caracterización de las CIM como constructo, donde se vislumbra el contexto teórico bajo el cual surgió, sus principales características y se hace un pequeño recuento de los autores más relevantes de este nuevo paradigma. Posteriormente, el trabajo se enfoca en la caracterización que da cuenta de cuál es su utilidad dentro de los tópicos de estudio de la gestión ambiental, y por qué es relevante su tratamiento por parte de las organizaciones. Para ello hace mención de manera diferenciada de sus principales instrumentos.

La segunda parte del trabajo, es de orden interpretativo. Tras la caracterización hecha de la CIM y de sus herramientas más comunes, se hace un análisis interpretativo de ésta desde una perspectiva ambiental, tomando como base los aportes de importantes autores contemporáneos, como Carabaza et.al (2007) y Saez (2005), entre otros. Autores que con perspectivas asertivas y pertinentes relacionadas con la atención y educación ambiental a través de la comunicación, sirven como marco de referencia para abordar la problemática propuesta.

El punto de partida para la detección de fuentes bibliográficas fue la revisión de diferentes journals del área. A partir de allí se pusieron de manifiesto los autores que han venido trabajando el tema. Se realizaron búsquedas con los siguientes parámetros: Integrated Marketing Communications, Green Advertising, Environmental Advertising, Green Marketing, Green Communications, adicionalmente de sus respectivas traducciones en idioma español.

Para dichas búsquedas se utilizaron: EBSCOhost Business Source Complete, Emerald, IEEE/IEE Electronic Library, JSTOR, Redalyc, Scielo, ScholarGoogle, ScienceDirect, Taylor \& Francis, y se revisaron diferentes revistas y publicaciones tanto en el idioma español como en inglés. La búsqueda se realizó para documentos publicados en el período comprendido entre 1993 y 2009, se tomaron las teorías relevantes y se revisaron las fuentes más importantes en estos artículos.

\section{Revisión conceptual}

La CIM es un concepto relativamente nuevo, que ha venido evolucionando con gran rapidez a lo largo de los últimos años. Es así como sus alcances hoy en día van más allá de lo que inicialmente se esperaría, pues partiendo del hecho de que su ámbito de aplicación se ha planteado desde un enfoque estratégico, muchos de sus elementos se han venido in- 
tegrando a cada una de las áreas de la organización de manera particular según se requiera, y de manera que ya no se piense en éste como un enfoque estrictamente del área de marketing, sino como parte del todo complejo que es la organización.

Uno de esos alcances es el ambiental, pues en la medida que los productos de la organización se han transformado a raíz de las nuevas necesidades ecológicas de los consumidores, la comunicación también ha tenido que adaptarse a este nuevo contexto.

Es por esto que resulta necesario entender la evolución de la CIM, pues a medida que el concepto ha venido evolucionando, nuevos enfoques y nuevas herramientas se han desarrollado para garantizar un mayor acercamiento con el eco-consumidor.

\subsection{Un acercamiento a la CIM como constructo}

En primer lugar, es importante reconocer la evolución, que como constructo, ha tenido la CIM, pues debemos partir del hecho que es un concepto complejo que no puede definirse desde una sola visión, y que por lo tanto hay que considerar desde distintas perspectivas para su posterior análisis.

Para comenzar, la definición de CIM utilizada por la Asociación Americana de Agencias de Publicidad AAAA (1989, citado por Duncan \& Everett, 1993) que la definió como "un concepto de comercialización planificación de las comunicaciones que reconoce el valor añadido de un plan integral que evalúa el papel estratégico de una variedad de comunicaciones disciplinas, por ejemplo, publicidad, respuesta directa, promoción de ventas y relaciones públicas--y combina estas disciplinas para brindar claridad, coherencia y comunicaciones máximo impacto." Esta es una primera gran idea de lo que se entiende por CIM, no obstante, existen enfoques algo más elaborados que permiten entender este constructo de una manera más integral entre la organización y su entorno.

Así por ejemplo, están los aportes realizados por Schultz (1991), Keegan et al. (1992), Kotler et al. (1999), Duncan (2002), Schultz \& Schultz (2004) y Kliatchko (2005), entre mucho otros, que muestran la evolución misma del concepto de CIM y en particular su significancia estratégica en las organizaciones. Valga la pena mencionar la validez de estos enfoques en relación con el objetivo de este documento, pues se dirigen concretamente al ámbito estratégico de la organización.

Deteniéndonos un momento en éstas definiciones podemos ver como por ejemplo Schultz (1991) define CIM como el proceso de gestión de todas las fuentes de información sobre un producto o servicio que se expone a un cliente o prospecto, que conductualmente mueve al cliente hacia una venta y logra mantener su fidelidad.

Otros enfoques interesantes son, por ejemplo, los planteados por Keegan et al. (1992), que define la CIM como la coordinación estratégica de todos los mensajes y los medios utilizados por una organización para influir colectivamente en el valor percibido de su marca.; o por Kotler et al. (1999), que se refiere a la CIM como el concepto bajo el cual una empresa integra y coordina cuidadosamente sus canales de comunicación con miras a entregar un mensaje claro, coherente y convincente sobre la organización y sus productos.

En el mismo sentido, autores como Duncan (2002) define la CIM desde la concepción de un proceso de funciones cruzadas para crear y nutrir las relaciones rentables con los clientes y otras partes interesadas, para controlar estratégicamente o influenciar todos los mensajes enviados a estos grupos y promover el diálogo propositivo con ellos a través del manejo de datos. Schultz \& Schultz (2004) hacen algo similar al referirse a la CIM como un proceso estratégico de negocios usado para planificar, desarrollar, ejecutar y evaluar coordinadamente, y medir los programas de comunicación persuasiva de la marca en el tiempo teniendo en cuenta a los consumidores, clientes, clientes potenciales, otros grupos concretos, y audiencias relevantes externas e internas.

Podemos también traer a colación enfoques más recientes como el de Kliatchko (2008), donde la CIM se entiende como el concepto y el proceso de administrar estratégicamente los programas de comunicación de marca centrados en audiencias focalizadas 
-lo que llamaríamos stakeholders-, el contenido, los canales, y orientados a los resultados a través del tiempo. O mejor aún, la definición propuesta por Shimp (2007), donde se destacan elementos como la planificación, creación, integración y puesta en práctica de diversas formas de comunicaciones de mercadeo (publicidad, promociones de ventas, ejecución de la publicidad, patrocinio de eventos, entre otros), el objetivo de influir o afectar directamente el comportamiento de la audiencia objetivo, y el cliente como punto de partida de la estrategia de comunicación de la organización.

Vemos entonces como, y de acuerdo con Kerr et al. (2008), esta redefinición continua de CIM refleja poco más de una década de investigación y la práctica del concepto en sí mismo. El creciente cuerpo de literatura se ha ocupado de cuestiones relativas a la definición del concepto, tales como explicaciones de la CIM, sus etapas (Keller, 2001) y la tipología de los mensajes, así como de cuestiones de aplicación, tales como la estructura, la responsabilidad de la organización, las actitudes hacia el enfoque de CIM y la integración de disciplinas. Kerr et al. (2008) menciona que como tal, la teoría de CIM en gran medida ha surgido a partir de observaciones de la práctica, según lo informado por una serie de investigadores como Duncan \& Everett (1993), Hartley \& Pickton (1999), Kitchen \& Schultz (1999), Low (2000), Swain (2004), entre otros.

No obstante, la ambigüedad sobre las cuestiones de definición y la falta de una base teórica, según Kerr et al. (2008) ha causado a muchos investigadores la pregunta de si CIM es un concepto nuevo en absoluto, o simplemente es una versión actualizada de lo que los profesionales de la comunicación siempre han tratado de hacer. De esta manera, basado en Cornelissen \& Lock (2000) y Gould, (2000), quienes afirman que si bien algunos investigadores señalan la falta de contenido académico y rigor de CIM, su simplificación y soluciones prescriptivas, y su uso de la retórica como justificación; otros afirman que esto no es raro en la gestión de muchos nuevos conceptos de marketing.

Ahora bien, para la visión de la CIM como una herramienta de marketing de largo alcance que vela por la sostenibilidad de la estrategia ambiental de las organizaciones, y que nos proponemos analizar en este documento, es necesario profundizar en dos aspectos fundamentales que justifican esta relación: el concepto de CIM desde la perspectiva ecológica -O ambiental - (que surge de las necesidades y expectativas de los eco-consumidores); y la caracterización de estos últimos y el reconocimiento de la incidencia de los demás stakeholders en las decisiones de la CIM ecológica que toman las organizaciones.

\subsection{La comunicación ecológica}

Según Calomarde (2000) citado en Lorenzo (2002), la comunicación ecológica deberá informar sobre los atributos de los productos, sobre sus beneficios para el medio ambiente y transmitir tanto una imagen de empresa bien informada y activa en temas ambientales como los mensajes a los posibles clientes sobre los ecoproductos. Así mismo también debe estar orientada a persuadir a los consumidores potenciales para que adquieran los productos ecológicos con preferencia, facilitando una adecuada información sobre sus beneficios, y también orientada a recordar el target, que estén familiarizados con los productos y sus ventajas, con el objetivo de que no se pierda la información entre el resto de los datos que les llega y que no pueden mantener indefinidamente en su mente.

Esto se logra entonces por medio de lo que en palabras de Shimp (2007) podemos identificar como Herramientas de la CIM: la publicidad, promoción de ventas, las relaciones públicas, y el patrocinio de eventos como principales instrumentos. Así las cosas, la meta de las estrategias de comunicación es crear una identidad corporativa clara y sólida para la compañía, aprovechando todos los recursos de comunicación que ésta tenga.

Es claro además que la mezcla de comunicación debe corresponderse con la imagen ecologista deseada. Las actividades de comunicación superficiales y a corto plazo nunca encontrarán credibilidad.

Los principales instrumentos de la comunicación, que según esta propuesta pueden apoyar exitosamente la ejecución de campañas de comunicación verde son entonces: 
- La publicidad: Para el caso de los productos ecológicos nunca debe ser una forma superficial de llevar adelante la defensa y conservación del medioambiente como un argumento más. Las campañas en defensa del medioambiente son muy complejas y requieren de una argumentación sostenida al largo plazo. Así, los anuncios, las campañas y demás ejecuciones publicitarias deben estar enfocadas a la comunicación de la realidad actual del producto y deben planearse con toda la rigurosidad pertinente para garantizar su impacto. La publicidad ecológica al ser compleja debe focalizarse en un posicionamiento cuidadoso y una acertada diferenciación de los productos sobre la base de la protección del medioambiente, así como el reconocimiento y el prestigio social. Puede decirse que las certificaciones medioambientales serían uno de los mejores ejemplos de publicidad verde, en la medida que cumple con todas éstas características y logra el fin último enfocado en la persuasión sobre la calidad ecológica de los productos que ofrece la organización.

- La promoción de ventas: Entendida como la utilización de técnicas de incentivo que crean una percepción de un valor mayor de la marca entre los consumidores, el comercio y los compradores, con el fin de incrementar en el corto plazo el nivel de ventas (O'guinn et al., 2007), adquiere una connotación de trabajo veloz, eficiente y convincente para los productos verdes. Esto se sustenta en acciones como el ofrecimiento de cupones con mensajes ecológicos, ofertas de rebajas en los precios de algunas existencias de productos ecológicos, o los concursos y sorteos defendidos a partir de la iniciativa por proteger el medio ambiente a partir de la compra de los productos de la empresa.

- Las relaciones públicas: Otro instrumento muy importante para la CIM verde, pues son aquellas actividades que buscan promover a la compañía en relación con los temas ecológicos. La única manera de recuperar y crear confianza en el público es poseer un buen sistema de relaciones públicas que sea coherente con los conceptos medioambientales, es decir, ir más allá de una imagen positiva de la compañía, debe despertar la conciencia y la sensibilidad de las distintas audiencias ofreciéndoles una información fiable.

Las acciones de relaciones públicas con enfoque ecológico deben tener un manejo muy especial. Las declaraciones que se den deben poder ser sometidas a cualquier tipo de pruebas, debe contribuir a la educación ambiental de la mayor cantidad de personas posibles, persuadir sobre los progresos de la empresa en todos los frentes en que se haya trabajado relacionados con el medioambiente; mencionar temas en materia de reciclaje, condiciones medioambientales de trabajo, cumplimiento de las normas legales, etc. Las relaciones públicas además deben realizarse a través de una campaña que sirva para fortalecer la conciencia ecológica, crear una cultura ecológica y una imagen de empresa competente en materia medioambiental.

Así mismo, las actividades de comunicación ejecutadas a través del canal de las relaciones públicas pueden ser defensivas, cuando se esfuerza por contrarrestar una imagen ecológicamente deficiente, y se difunde información sobre sus logros en el campo de la protección del medioambiente haciendo énfasis en su responsabilidad social; u ofensivas, cuando se ve en el desafío ecológico una oportunidad para crear potencialidades de éxito a largo plazo en el mercado, y se hacen muchos esfuerzos para fortalecer la conciencia ecológica y responder a las exigencias de los consumidores a largo plazo.

- Patrocinio de eventos: Estos deben desarrollarse a través de instrumentos clásicos pero enfocados al tema medioambiental, tales como conferencia de prensa y boletines, seminarios sobre medioambiente, obras benéficas relacionadas con el medioambiente, entre otras actividades; que integradas con una buena gestión de relaciones públicas puede contribuir con el posicionamiento de la imagen de marca como ecologista o a favor del medio ambiente. 
Ahora bien, es importante analizar la importancia que han tenido las CIM en el desarrollo de programas de marketing verde en las organizaciones, y de ésta manera ampliaremos un poco la reflexión sobre la perspectiva real del problema de comunicación presente en las empresas que desarrollan productos amigables con el medio ambiente.

\subsection{Implicaciones estratégicas de la CIM verde en el contexto actual}

Investigaciones como las de Hartmann \& ApaolazaIbáñez (2009) dan apoyo a la hipótesis de que, en sociedades en las que las experiencias con la verdadera naturaleza son cada vez más escasas, el consumo de productos verdes -acondicionado de forma adecuada con experiencias virtuales en la naturaleza - puede servir como un sustituto de un "verdadero" contacto con la naturaleza. De este modo, cuando imágenes de la naturaleza o de un paisaje agradable se presentan junto con material informativo sobre reclamaciones ambientales de los consumidores, se garantiza la eficacia de la publicidad verde.

Se ve como se trata no sólo de querer llevar un mensaje a unas audiencias específicas, sino además de hacerlo de una manera efectiva, aprovechando las herramientas que mayor contacto nos garanticen con las comunidades que están involucradas con nuestro producto o servicio; y sobretodo atacando esa necesidad por lo ambiental y lo ecológico que la sociedad de hoy reclama a gritos como resultado de su alejamiento a actividades que impliquen el contacto con el medio natural.

A pesar de esta necesidad, es notable la problemática que citan autores como Carabaza et.al, (2007), relacionada con la falta de atención en la temática ambiental en la agenda mediática nacional de algunos países latinoamericanos. Mencionan lo preocupante que resulta que los medios de comunicación no se encuentren abordando todos los macro temas y temas que tienen que ver con la problemática ambiental, ya que la sociedad en general, o al menos las audiencias que se exponen frecuentemente a estos medios (en este caso, la televisión), no alcanzan a percibir la magnitud de ésta problemática; y por lo tanto a comprender que ésta, además de estar fuertemente relacionada con la naturaleza, tiene implicaciones mayores que están impactando en los fenómenos climáticos y en la salud.

Así las cosas, la comunicación en el marketing verde va más allá de las implicaciones operativas de su aplicación; pues la profundidad de sus contenidos y los objetivos que la caractericen son las variables que garantizarán su efectividad. Efectividad que debe medirse no sólo con el alcance de las campañas -número de personas a las que llega el mensaje en un determinado espacio de tiempo (O'guinn, et al., 2007)-, o el rating que se obtenga por cada pauta o anuncio; sino a través de los resultados en cambios en el comportamiento y educación de las audiencias a las cuáles se les están dirigiendo los mensajes.

Se comparte entonces desde este documento la postura de Sáez (2005), cuando afirma que para que la educación ambiental, mediante los medios de comunicación masivos, desempeñe un verdadero papel educativo es necesario que tenga un correcto enfoque filosófico, sociológico, y psicopedagógico y así aprovechar al máximo las potencialidades de estos medios y que no se convierta el mensaje ambiental en "pura publicidad" sino en educación, es decir, que modifique la forma de pensar y actuar de los individuos.

Valga entonces ampliar esta propuesta para todos los medios de comunicación por los cuales está llegando el mensaje a las audiencias objetivo, es decir, no solo habría que pensar esta situación desde los medios masivos, sino desde todos aquellos posibles canales de comunicación que utiliza la organización para persuadir sobre sus actividades ambientales.

Así mismo, no debe olvidarse que la comunicación planteada desde el marketing verde debe informar según los objetivos ecológicos planteados por la organización. No solo se trata de comunicar los atributos del producto, sino también de persuadir a los consumidores potenciales sobre las ventajas de los productos ecológicos, sobre los no ecológicos, y proporcionar valor agregado al cliente y a los grupos de interés a través de la información suministrada. 


\subsection{Audiencias de la CIM ecológica}

\subsubsection{El consumidor verde}

De acuerdo con Chamorro et al. (2005), el principal tópico de investigación en el área del marketing verde es el denominado "consumidor ecológico", pues en los últimos años se ha presentado un notable crecimiento en el número de publicaciones sobre este tópico que lo convierten en uno de los de mayor proyección para la próxima década. Así las cosas, no será en vano referirnos a las características más relevantes del eco-consumidor, las cuáles son tal vez el foco del desarrollo de la comunicación y demás acciones en favor del enfoque medioambiental que hoy tienen las organizaciones.

Es así, como en relación con postulados como los de Zinkhan \& Carlson (1995), puede decirse que muchos consumidores en la actualidad se han convertido en consumidores que presentan preocupaciones por algo más que la compra y los procesos de consumo. También están preocupados por el proceso de producción, en términos de los escasos recursos consumidos, y adicionalmente analizan problemas como la eliminación de productos y el reciclaje.

Es por esto que en palabras de Durmaz \& Zergin (2011), existe un interés creciente entre los consumidores de todo el mundo en materia de protección del medio ambiente. Pruebas en todo el mundo indican que las personas están preocupadas por el medio ambiente y están cambiando su comportamiento. De aquí que muchas organizaciones hayan optado por dar importante énfasis en las preocupaciones del llamado consumidor verde (Stafford et al., 1996).

Así, puede verse cómo el número de consumidores ecológicos crece, y como resultado, las organizaciones reconocen que estos individuos pueden ser suficientes como para crear un segmento de mercado amplio y viable. Por lo tanto, las organizaciones pueden poner en práctica estrategias de marketing verde porque les resulta rentable hacerlo. Las organizaciones pueden tomar decisiones con enfoque ambiental porque se han dado cuenta de que un segmento de la base de clientes es ecológico.
Sin embargo, y por desgracia para las organizaciones, los consumidores verdes también son muy susceptibles de albergar sesgos anti-corporativos; pues son el segmento que probablemente más desconfía de los anunciantes y por lo tanto son muy proclives a seguir conductas y actividades que confunden a los directores de las organizaciones, dentro de los cuales claramente están los directores de comunicación (Zinkhan \& Carlson, 1995).

\subsubsection{Stakeholders verdes}

Para Kliatchko (2009) el término stakeholders -en la teoría de CIM- se refiere a los múltiples mercados o públicos relevantes de una empresa y, más concretamente, a los distintos mercados hacia los cuales están dirigidos sus productos y servicios. Esto incluye los públicos tanto internos como externos. Un profundo conocimiento de sus consumidores con necesidades, valores, motivaciones, y lo más importante, su comportamiento en el mercado es un paso fundamental y vital en la realización de un programa de CIM. Por lo tanto resultará fundamental, para la comprensión de este documento, identificar la importancia de estos grupos en el enfoque estratégico ambiental.

Es por lo anterior que, a partir de los planteamientos de Zinkhan \& Carlson (1995) puede afirmarse que así como los consumidores pueden ser verdes, los propietarios (o sus empleados o proveedores) también pueden serlo. Así por ejemplo, muchas organizaciones son participes de agremiaciones que se componen enteramente de empresas que cumplen ciertos estándares de responsabilidad corporativa, y específicamente medioambientales; y que adicionalmente se esfuerzan por presentar la etiqueta verde dentro de sus labores de comunicación, que les permitirán atraer inversores y relacionarse con otros grupos que pueden resultar interesantes para llevar a cabo sus proyectos de gestión.

De acuerdo con este enfoque, es de esperarse que un gestor cuidadoso propenda por una estrategia verde para el propio bien de la organización, pues finalmente esto es lo que hay que hacer. En otras 
palabras, es mucho más importante hacer lo correcto (en un sentido moral) que hacer las cosas bien (en un sentido técnico). Para tal fin, el marketing y la comunicación verde, florecerán y darán importantes frutos. De este modo, los stakeholders en cuestión (tales como los proveedores o las entidades reguladores gubernamentales) también pueden incentivar a una organización hacia la aplicación de estrategias verdes.

Es entonces, como el desarrollo de los fines organizacionales es visto desde la perspectiva responsable, planteando las prácticas ambientales, tales como el desarrollo de productos verdes, la iniciativa más conveniente en materia de adaptación a las necesidades de los consumidores actuales. No obstante, estas prácticas requieren ser comunicadas y difundidas a todos los grupos de interés de la organización, pues son estos grupos quienes se reconocerán con posteridad como las audiencias de la comunicación verde $y$, que por lo mismo, establecerán los requerimientos en materia de comunicación que deben cumplir las empresas para ser escuchadas y reconocidas como una unidad ejecutora de acciones en favor de la ecología y el medio ambiente.

Calomarde (2000) citado por Lorenzo (2002) plantea que mediante la comunicación se proporciona un valor agregado al cliente: la información. Los destinatarios de este tipo de comunicación son todos los grupos de interés, tanto internos como externos, y no sólo el consumidor. Además, los reclamos racionales desarrollan un papel muy importante en el proceso de decisión de compra y puesto que lo que se pretende es que el estímulo que provocan en el consumidor sea duradero en el tiempo y que penetre profundamente en la mente, es imprescindible una comunicación intensiva respecto a los productos equivalentes no ecológicos.

Es entonces visible, la importancia de generar una comunicación verde con objetivos definidos como propios, diferenciados de los objetivos generales de mercadeo, que permitan medir y evaluar los resultados de las campañas que se realizan para cada audiencia.

\section{Conclusiones}

El mercadeo verde puede entenderse como el resultado de la reacción de las organizaciones a la evolución de un mercado cada vez más exigente, con consumidores que reconocen la importancia de los productos amigos del medioambiente y por ende de las empresas que producen estos.

El compromiso entonces de las organizaciones, se tiene que traducir en un modelo estratégico de mercadeo desde el que es posible conseguir al mismo tiempo la satisfacción de las necesidades de los consumidores, la maximización de los beneficios de la empresa y la minimización de los impactos negativos sobre el medio ambiente en la actividad de intercambio.

Para lograr hacer efectiva esta nueva propuesta de mercadeo verde las dos últimas décadas, se han creado iniciativas desde la perspectiva estratégica de la Comunicación Integrada de Marketing (CIM) que propenden por cambiar la forma de proceder, la visión y el actuar de las personas. Perspectiva que valida el interés estratégico de la Comunicación y presenta la integración como el camino a la difusión adecuada de mensajes a las distintas audiencias de la organización.

Estas nuevas estrategias e innovaciones en la comunicación, requieren el desarrollo de mensajes efectivos que tengan un balance entre la identificación de las necesidades del eco-consumidor, la calidad de los productos ecológicos ofrecidos por la empresa, el desempeño de los canales de comunicación utilizados y la medición de su impacto en las audiencias a las que van dirigidos.

Las conceptualizaciones de CIM, y en particular las aplicaciones en la gestión ambiental estudiadas aquí, evalúan unas el proceso y otras el resultado o intentan hacer una integración de los dos.

Si se orienta la evaluación de la CIM dentro de la gestión ambiental hacia el proceso, más que hacia el resultado, esto supone admitir que la determinación de ésta en las organizaciones debe estar basada fun- 
damentalmente en las percepciones que los consumidores y demás audiencias tienen sobre el producto o servicio, tal como lo plantean autores como Chan (1999) y Chan \& Lorett $(2002,2004)$.

Si se orienta la evaluación hacia el resultado, más que hacia el proceso, esto supone que la determinación de la comunicación sobre los productos se basa no en las percepciones que los consumidores verdes tienen sobre éste, sino en qué tan eficaz es la organización para satisfacer las necesidades de los mismos, específicamente la expectativa general con respecto a su calidad de ecológicos, lo cual es demasiado complejo de medir, si se toma como base la evidencia de que una certificación ya no es suficiente para comprobar esta característica.

No obstante, responder a los requerimientos nacientes y en aumento de una sociedad cada día más consciente de sus compromisos ambientales, requiere indiscutiblemente hacer una inversión significativa no solo en investigación y desarrollo, en certificaciones, sistemas de gestión ambiental; sino también en los medios y canales de difusión de la información relacionada con éstas actividades que se están desarrollando en pro del cuidado ambiental. Este documento brinda entonces un resumen de los instrumentos comunicativos que pueden ayudar a lograr este objetivo y cuáles son los requerimientos de las audiencias que deben tenerse en cuenta.

Adicionalmente, es importante considerar la CIM como una fuente de oportunidades para incrementar la competitividad de las organizaciones, a partir del nuevo enfoque estratégico propuesto desde las teorías del marketing y aprovechándola en la obtención de objetivos de difusión de los logros ambientales obtenidos por la organización.

Con respecto a las futuras líneas de investigación, queda el camino abierto a nuevos aportes con respecto a los alcances que pueda tener la CIM como medio estratégico de difusión de los mensajes medioambientales, y las implicaciones que puedan generarse de su utilización en las organizaciones de hoy. Se propone entonces la profundización en el estudio de cada uno de los instrumentos de CIM, con miras a lograr objetivos de impacto ambiental; pues si bien se han identificado algunas de las fortalezas de cada instrumento, se ve la limitación de este documento en cuanto al tratamiento profundo de los mismos. Por lo tanto se considera que éste puede ser un gran tema de investigación, particularmente acerca de los beneficios puntuales que puede tener la utilización de cada una de estas herramientas en la estrategia de comunicación ambiental, y cómo un instrumento podría verse como más conveniente que otro para apoyar la misma en un contexto específico.

\section{Referencias}

Carabaza, J., Lozano, J., González, J., Pasco L., Reyes, L., Berumen, A. \& Álvarez, P. (2007). Cobertura Del Medio Ambiente en la Televisión Mexicana. En: Revista Comunicación y Sociedad, Universidad de Guadalajara, Enero-Junio, 7: 45-76.

Chamorro, A., Miranda, F. \& Rubio, S. (2005). La investigación Académica en Marketing Ecológico: Diferencias entre las Publicaciones Españolas y las Internacionales. XVII Encuentro de profesores universitarios de marketing. Madrid, 22-23 de septiembre de 2005. ISBN 84-7356-424-3, pp. 883-902.

Chan, R. (1999). Environmental Attitudes and Behavior of Consumers in China. En: Journal of International Consumer Marketing, 11(4): 25-52

Chan, R. \& Lau, L. (2002). Explaining Green Purchasing Behavior -A Cross-Cultural Study on American and Chinese Consumers. En: Journal of International Consumer Marketing, 14(2-3): 9-40.

Chan, R. \& Lau, L. (2004). The Effectiveness of Environmental Claims among Chinese Consumers: Influences of Claim Type, Country Disposition and Ecocentric Orientation. En: Journal of Marketing Management, 20(3-4): 273-319.

Cornelissen, J. \& Lock, A. (2000). Theoretical concept or management fashion. Examining the significance of IMC. En: Journal of Advertising Research, 40(5): 7-15.

Duncan, T. (2002). IMC: Using Advertising and Promotion to Build Brands. International Edition, The McGraw-Hill Companies.

Duncan, T. \& Everett, S. (1993). Client Perceptions Of Integrated Marketing Communications. En: Journal of Advertising Research, May/Jun, 33( 3): 30-39.

Durmaz, Y. \& Zergin, S. (2011). Theoretical Approach to Concept Of Green Marketing. En: Interdisciplinary Journal of Contemporary Research in Business, 3 (2): 1808-1814.

Gould, S. (2000). The state of IMC research and applications. En: Journal of Advertising Research, 40(5): 22-23.

Hartley, B. \& Pickton, D. (1999). Integrated Marketing Communications require a New way of thinking. En: Journal of marketing Communications, 5(2): 97-106

Hartmann, P. \& Apaolaza-Ibáñez, V. (2009). Green Advertising Revisited. Conditioning Virtual Nature Experiences. En: International Journal of Advertising, 28(4): 715-739.

Keegan, W., Moriarty, S. \& Duncan, T. (1992). Marketing. PrenticeHall, Englewood Cliffs, NJ.

Keller, K. (2001). Mastering the Marketing Communications Mix: Micro and Macro Perspectives on Integrated Marketing Com- 


\section{LAS COMUNICACIONES INTEGRADAS DE MARKETING (CIM) COMO PILAR DE LA ESTRATEGIA DE MARKETING VERDE Y SUS IMPLICACIONES EN LA GESTIÓN AMBIENTAL}

munication Programs. En: Journal of Marketing Management, 17: 819-847.

Kerr, G., Schultz, D., Patti, Ch. \& Kim, I. (2008). An inside-out approach to integrated marketing communication, an international analysis. En: International Journal of Advertising, 27(4): 511-548.

Kitchen, P. \& Schultz, D. (1999). A Multi-Country Comparison of the Drive for IMC. En: Journal of Advertising Research, 39 (1): 21-38.

Kliatchko, J. (2005). Towards a New Definition of Integrated Marketing Communications (IMC). En: International Journal of Advertising, 24(1): 7-34.

Kliatchko, J. (2008). Revisiting the IMC construct- A revised definition and four pillars. En: International Journal of Advertising, 27(1): 133-160.

Kliatchko, J. (2009). IMC 20 Years After: A Second Look at IMC Definitions. En: Intemational Journal of Integrated Marketing Communications, 1(2): 7-12.

Kotler, P., Armstrong, G., Saunders, J. \& Wong, V. (1999). Principles of Marketing. (2nd European edition). London: Prentice Hall Europe.

Lorenzo, M. (2002). Marketing Ecológico y Sistemas de Gestión Ambiental: Conceptos y Estrategias Empresariales. En: Revista Galega de Economía, 11 (2): 1-25.

Low, G. (2000). Correlates of integrated marketing communications. En: Journal of Advertising Research, 40 (3): 27-39.

Madhavaram, S., Badrinarayanan V. \& McDonald, R. (2005). Integrated Marketing Communication (IMC) and Brand Identity as Critical Components of Brand Equity Strategy. En: Journal of Advertising, 34(4): 69-80.
O'guinn, T., Allen, C. \& Semenik, R. (2007). Publicidad y comunicación integral de marca. $4^{\mathrm{a}}$ edición. Ed. Cengage Learning México.

Saez, C. (2005). Los Spots, una posibilidad para desarrollar la educación ambiental a través de la televisión. En: Revista Comunicar, $N^{\circ} 25$. Grupo Comunicar, Huelva - España.

Schultz, D. (1991). Integrated Marketing Communications: The Status of Integrated Marketing Communications Programs in the US. En: Journal of Promotion Management, 1: 37-41.

Schultz, D. \& Kitchen, P. (2000). A response theoretical concept or management fashion? En: Journal of Advertising Research, 40(5): $17-21$

Schultz, D. \& Schultz, H. (2004). IMC The next generation. Five steps for delivering value and measuring returns using marketing communications. McGraw-Hill Nueva York.

Shimp, T. (2007). Integrated marketing communications in advertising and promotion. Ed. South-Western Cengage Learning, USA.

Stafford, M., Stafford, T. \& Chowdhury, L. (1996). Predispositions toward green issues: The potential efficacy of advertising appeals. En: Journal of Current Issues and Research in Advertising, 18 (1): 67-79.

Swain, W. (2004). Perceptions of IMC after a decade of development: Who's at the wheel, and how can we measure success? En: Journal of Advertising Research, 44 (1): 46-65.

Vélez, M. (2001). Situación actual en el campo del comercio de productos y servicios de la biodiversidad en la región andina. Ginebra: UNCTAD.

Zinkhan, G. \& Carlson, L. (1995). Green Advertising and the Reluctant Consumer. En: Journal of Advertising, 24 (2): 1-6. 